1999-03-15

\title{
Monte Carlo study of the capacitance of the double layer in a model molten salt
}

Douglas Henderson

Dezso Boda

Kwong-Yu Chan

Follow this and additional works at: https://scholarsarchive.byu.edu/facpub

Part of the Biochemistry Commons, and the Chemistry Commons

\section{Original Publication Citation}

Boda, Dezso, Douglas Henderson, and Kwong Y. Chan."Monte Carlo study of the capacitance of the double layer in a model molten salt." The Journal of Chemical Physics 11 (1999): 5346-535.

\section{BYU ScholarsArchive Citation}

Henderson, Douglas; Boda, Dezso; and Chan, Kwong-Yu, "Monte Carlo study of the capacitance of the double layer in a model molten salt" (1999). Faculty Publications. 626.

https://scholarsarchive.byu.edu/facpub/626 


\title{
Monte Carlo study of the capacitance of the double layer in a model molten salt
}

\author{
Dezsö Boda ${ }^{a)}$ and Douglas Henderson ${ }^{b)}$ \\ Department of Chemistry and Biochemistry, Brigham Young University, Provo, Utah 84602-5700 \\ Kwong-Yu Chan ${ }^{\mathrm{c})}$ \\ Department of Chemistry, The University of Hong Kong, Pokfulam Road, Hong Kong
}

(Received 29 October 1998; accepted 15 December 1998)

\begin{abstract}
Monte Carlo simulations are reported for charged hard spheres at high density near a charged wall. This system is a simple model for a molten salt double layer. Unfortunately, the reduced temperatures that correspond to experiment are very small. This results in a large Boltzmann factor. As a result, we are unable to obtain meaningful results for such low values and report results only for moderately low values of the reduced temperature. Even so, our results should be a useful benchmark. Further, we are able to give a qualitative answer to an interesting question. We find that at low temperatures the capacitance near the point of zero charge increases with increasing temperature. This agrees with experiment for molten salts and disagrees with the behavior of double layer in dissolved salts, which can be modeled with low density and high temperature charged hard spheres near a wall. This also disagrees with the predictions of the Gouy-Chapman theory and the mean spherical approximation. It appears that it is the approximations, and not the charged hard sphere double layer model, that are at fault for describing double layers in molten salts. (C) 1999 American Institute of Physics. [S0021-9606(99)51011-8]
\end{abstract}

\section{INTRODUCTION}

A long standing puzzle in theoretical electrochemistry is the fact that the capacitance near the point of zero charge of a double layer (DL) formed in a molten salt/electrode interface increases with increasing temperature. This is different from the situation in DLs that are formed in aqueous electrolytes and other solvents, where the capacitance decreases with increasing temperature.

Experimentally, it is the differential capacity, $d \sigma / d \Psi_{0}$, that is measured. However, near the point of zero charge, where the potential, $\Psi_{0}$, is a linear function of the charge density of the electrode, $\sigma$, the differential and integral capacitance, $\sigma / \Psi_{0}$, are equal. In this paper, we will confine ourselves to small $\sigma$ and will not distinguish between the differential and integral capacitance, and will use only the word capacitance.

Theory has no difficulty in making a prediction for the temperature dependence of the capacitance of a dissolved salt DL. For example, in the linearized Gouy-Chapman (GC) theory

$$
C=\frac{\epsilon}{4 \pi} \kappa,
$$

where $\epsilon$ is the dielectric constant of the medium in which the ions are located and $\kappa$ is the inverse Debye screening length,

\footnotetext{
${ }^{a}$ Permanent address: Department of Physical Chemistry, University of Veszprém, H-8201 Veszprém, PO Box 158, Hungary. Electronic mail: boda@almos.vein.hu

${ }^{\text {b)} E l e c t r o n i c ~ m a i l: ~ d o u g @ h u e y . b y u . e d u ~}$

${ }^{c}$ Electronic mail: hrsccky@hku.hk
}

$$
\kappa^{2}=\frac{4 \pi \beta\left(z_{e} e\right)^{2} \rho}{\epsilon}
$$

where $z_{e} e$ is the magnitude of the ionic charge, $e$ is the magnitude of the charge of the electron, $\beta=1 / k T, k$ is the Boltzmann constant and $T$ is the temperature, and $\rho=N / V$ is the number density, where $N$ is the total number of positive and negative ions, and $V$ is the volume. For simplicity, we have assumed that the salt is symmetric. From Eqs. (1) and (2) it follows that

$$
\frac{d \ln C}{d \ln T}=-\frac{1}{2} .
$$

The GC results given above are based on the assumption that the ions are point charges. If it is assumed that the ions can approach only half an ion diameter from the electrode, as suggested by Stern, the linearized GCS result, corresponding to Eq. (3) is

$$
\frac{d \ln C}{d \ln T}=-\frac{1}{2\left(1+\frac{\kappa d}{2}\right)},
$$

where $d$ is the ion diameter. Painter et al. ${ }^{1}$ and Ballone $e t a l{ }^{2}$ have investigated the applicability of the mean spherical approximation (MSA) to molten salt DLs. The MSA treats the ionic diameter in a consistent manner whereas the GCS theory considers the ionic diameter only in the electrode-salt interaction. One would assume that the MSA would be an improvement over both GC and GCS treatments. The MSA expressions corresponding to Eqs. (1) and (3) are 
TABLE I. Results of the simulations and the MSA for the DL of molten salts. The partial densities, labeled as "left" and "right," are the contact values at the left and right wall. The density labeled with "middle" is the average reduced density in the middle of the cell, obtained by integrating the density profiles from $z=5 d$ to $z=7 d$. The densities included in the table are subject to a statistical uncertainty of $1-5$ in the last digits. The number of anions is $N_{-}$ $=235 ; 3$ million configurations were run for each state.

\begin{tabular}{|c|c|c|c|c|c|c|c|c|c|c|c|c|}
\hline$q^{*}$ & $T^{*}$ & $-\sigma^{*}$ & $N_{+}$ & $\begin{array}{c}-\Psi_{0}^{*} \\
\mathrm{MC}\end{array}$ & $\begin{array}{l}-\Psi_{0}^{*} \\
\text { MSA }\end{array}$ & $\begin{array}{c}C^{*} \\
\mathrm{MC}\end{array}$ & $\begin{array}{c}C^{*} \\
\text { MSA }\end{array}$ & $\begin{array}{l}\rho_{+}^{*} \\
\text { left }\end{array}$ & $\begin{array}{c}\rho_{+}^{*} \\
\text { right }\end{array}$ & $\begin{array}{l}\rho_{-}^{*} \\
\text { left }\end{array}$ & $\begin{array}{c}\rho_{-}^{*} \\
\text { right }\end{array}$ & $\begin{array}{c}\rho_{0}^{*} \\
\text { middle }\end{array}$ \\
\hline 1.2004 & 0.8331 & 0.02344 & 238 & 0.151 & 0.175 & 0.187 & 0.134 & 1.583 & 1.592 & 1.360 & 1.357 & 0.636 \\
\hline 2.4008 & 0.4165 & 0.02344 & 238 & 0.103 & 0.139 & 0.228 & 0.169 & 1.588 & 1.578 & 1.282 & 1.284 & 0.636 \\
\hline \multirow[t]{4}{*}{3.5472} & 0.2819 & 0.02344 & 238 & 0.087 & 0.122 & 0.270 & 0.192 & 1.557 & 1.559 & 1.213 & 1.211 & 0.638 \\
\hline & & 0.03906 & 240 & 0.151 & 0.203 & 0.258 & 0.192 & 1.706 & 1.699 & 1.123 & 1.111 & 0.640 \\
\hline & & 0.07812 & 245 & 0.323 & 0.405 & 0.242 & 0.193 & 2.110 & 2.110 & 0.907 & 0.906 & 0.646 \\
\hline & & 0.11719 & 250 & 0.477 & 0.605 & 0.246 & 0.194 & 2.556 & 2.553 & 0.716 & 0.772 & 0.653 \\
\hline \multirow[t]{3}{*}{4.7297} & 0.2114 & 0.02344 & 238 & 0.110 & 0.111 & 0.212 & 0.211 & 1.526 & 1.525 & 1.118 & 1.124 & 0.644 \\
\hline & & 0.04688 & 241 & 0.219 & 0.221 & 0.214 & 0.212 & 1.782 & 1.787 & 0.966 & 0.973 & 0.647 \\
\hline & & 0.07031 & 244 & 0.328 & 0.331 & 0.215 & 0.212 & 2.065 & 2.074 & 0.822 & 0.831 & 0.652 \\
\hline 5.6670 & 0.1764 & 0.02344 & 238 & 0.116 & 0.105 & 0.203 & 0.224 & 1.544 & 1.542 & 1.089 & 1.083 & 0.644 \\
\hline \multirow[t]{4}{*}{7.0945} & 0.1410 & 0.01562 & 237 & 0.087 & 0.065 & 0.180 & 0.240 & 1.503 & 1.518 & 1.148 & 1.154 & 0.642 \\
\hline & & 0.02344 & 238 & 0.142 & 0.098 & 0.165 & 0.240 & 1.621 & 1.616 & 1.076 & 1.081 & 0.643 \\
\hline & & 0.03906 & 240 & 0.202 & 0.162 & 0.193 & 0.241 & 1.859 & 1.851 & 0.945 & 0.943 & 0.646 \\
\hline & & 0.07812 & 245 & 0.338 & 0.324 & 0.231 & 0.241 & 2.522 & 2.507 & 0.645 & 0.642 & 0.652 \\
\hline
\end{tabular}

$$
C=\frac{\epsilon}{2 \pi} \Gamma
$$

and

$$
\frac{d \ln C}{d \ln T}=-\frac{1+\Gamma d}{2(1+2 \Gamma d)},
$$

where $\Gamma$ is a renormalized screening parameter that is related to $\kappa$ by

$$
\kappa=2 \Gamma(1+\Gamma d) .
$$

From Eq. (6), we see that the MSA capacitance also decreases with increasing temperature, as was pointed out earlier by the above authors. ${ }^{1,2}$ The linearized GC and MSA theories do not distinguish between the differential and integral capacitance.

Thus, we are left with the question of whether it is the charged hard sphere model or the above approximations that are at fault. In this paper we have attempted to answer this question by means of Monte Carlo (MC) simulations.

In addition to answering this question, we hope that this will provide a useful benchmark for testing theories of molten salt DL. Li and $\mathrm{Mazo}^{3}$ have pointed out the need for such simulations.

\section{SIMULATIONS}

Monte Carlo simulations were performed for mixtures of $N_{+}$positively and $N_{-}$negatively charged hard spheres in a rectangular cell of dimensions $L \times L \times H$ at a temperature $T$. Hard, impenetrable walls are placed at $z=0$ and $z=H$, while periodic boundary conditions are applied in the $x$ and $y$ directions. Each wall carries the reduced surface charge

$$
\sigma^{*}=\frac{\sigma d^{2}}{e}=-\frac{z_{e}}{2}\left(\frac{d}{L}\right)^{2}\left(N_{+}-N_{-}\right) .
$$

Since we are studying the temperature dependence, the reduced temperature $T^{*}=d / \beta e^{2}$ is more appropriate for our purpose as an independent variable of the system. Note that this is the reciprocal of the reduced charge used by Torrie and Valleau, ${ }^{4}$ as well as Larsen, ${ }^{5} q^{*}=1 / T^{*}$, that is the more common choice of a variable. Further, note that both $\epsilon$ and $z_{e}$ are taken to be 1 in our simulations. The reduced density is defined as $\rho^{*}=\rho d^{3}$.

The long range corrections of the Coulombic forces were taken into account by the charged sheet method described in detail in our previous paper where we investigated the DL of an ion-dipole mixture. ${ }^{6}$ This is an adaptation of the method of Torrie and Valleau. ${ }^{4}$ To each ion there corresponds a charged sheet carrying the surface charge $e / L^{2}$ with a square hole in the middle, according to the simulation cell. The long range correction is obtained from the interaction between an ion and the sheet with a square hole, representing the image charges outside the cell. Note that the sheets are moving with ions in the simulation; therefore, the energy change between two consecutive states depends only on these states. Consequently, this simulation is rigorously a Markov chain, in contrast to the sheet method used by Torrie and Valleau ${ }^{4}$ for the simulation of the primitive model of the DL, because they used an averaged density profile for charge densities of equidistant sheets.

For simplicity we consider some of the values of $\eta$ $=\pi \rho d^{3} / 6$ and $q^{*}$ that Larsen ${ }^{5}$ has suggested are appropriate for molten salts. The states for which we report results are listed in Table I. Table I contains the reduced mean electrostatic potential drop across the double layer obtained from both the simulations and the MSA. The reduced potential is $\Psi^{*}=\Psi d / e$, and it is calculated from the integration of the charge density profiles in the usual way.

In the simulations, the number of particles and the volume were chosen so that the packing fraction was one of Larsen's ${ }^{5}$ values, $\eta=0.3503$. The values of $N=N_{+}+N_{-}$are 
given in Table I, and the volume used in computing $\eta$ was $L^{2}(H-d)$. When the surface charge on the walls were increased, the number of cations in the cell were increased, while the number of anions were kept fixed. This resulted in a slight increase in the average reduced density in the middle of the cell as seen in Table I, but it is small because the excess cations are taken mainly by the DL.

To characterize the capacitance, we introduced the reduced capacitance, $C^{*}=\sigma^{*} / \Psi_{0}^{*}$. Table I shows the values of the capacitance obtained from the simulations and the MSA. The MSA results for the potential and the capacitance are calculated using the corresponding $\rho_{0}^{*}$, the bulk density yielded by the simulation in the middle of the cell, in order to make a correct comparison.

Table I also contains the contact values of the densities of the cations and the anions at left and the right walls. Since the cell is symmetrical, the contact values for a given particle should be the same at the two walls. The consistency of these values is a measure of the accuracy of the simulations. The data in Table I show that the agreement of the left and right contact values is satisfactory.

Unfortunately, when $T^{*}$ is very low $\left(T^{*}<0.141\right)$ we found that the ions tended to form chain-like structures along the $z$-axis of the simulation cell. These chains are very similar to those obtained in our simulations for ion-dipole mixtures that model DL in solvents. ${ }^{6}$ The system was frozen into low energy structures of particles ordered into chains or clusters, and cannot be moved out of it in a reasonable time of simulation. The expression "practically nonergodic" was used by Larsen and Rogde ${ }^{7}$ for this behavior.

This is a bit of disappointment as the values of $T^{*}$ that are given in Table I are still too high for molten salts. As a result, we cannot make a direct comparison with experiment. Perhaps, with heroic efforts we could obtain results for lower $T^{*}$. However, it did not seem worthwhile to do so. Even so, these results are useful and some conclusions can be drawn.

The dimensions of the simulation cell were chosen to be $L=8 d$ and $H=12 d$. The chains mentioned above appear if the temperature or $L$ is too small, or if $\sigma^{*}$ or the density is too high. For instance, we were able to obtain good results for $L=6$ at $T^{*}=0.282$ but not at $T^{*}=0.141$. Using $L=8$, the chains disappeared, but by decreasing $T^{*}$ they appeared again.

A few simulations were performed for a higher density, $\eta=0.3945$. For $T^{*}=0.211$ we obtained almost the same capacitances than at $\eta=0.3505$. This implies that the density dependence of the capacitance is low. For $T^{*}=0.141$ the cell length $L=8$ proved to be too small, and chains were obtained.

\section{RESULTS}

Figure 1 shows normalized density profiles (they are normalized with the values for the densities in the middle of the cell tabulated in Table I) at a fixed temperature for different surface charges, while Fig. 2 gives the profiles at a fixed surface charge for various temperatures. The profiles look quite reasonable. At lower temperatures and at higher surface charges, stronger layering is found near the walls.

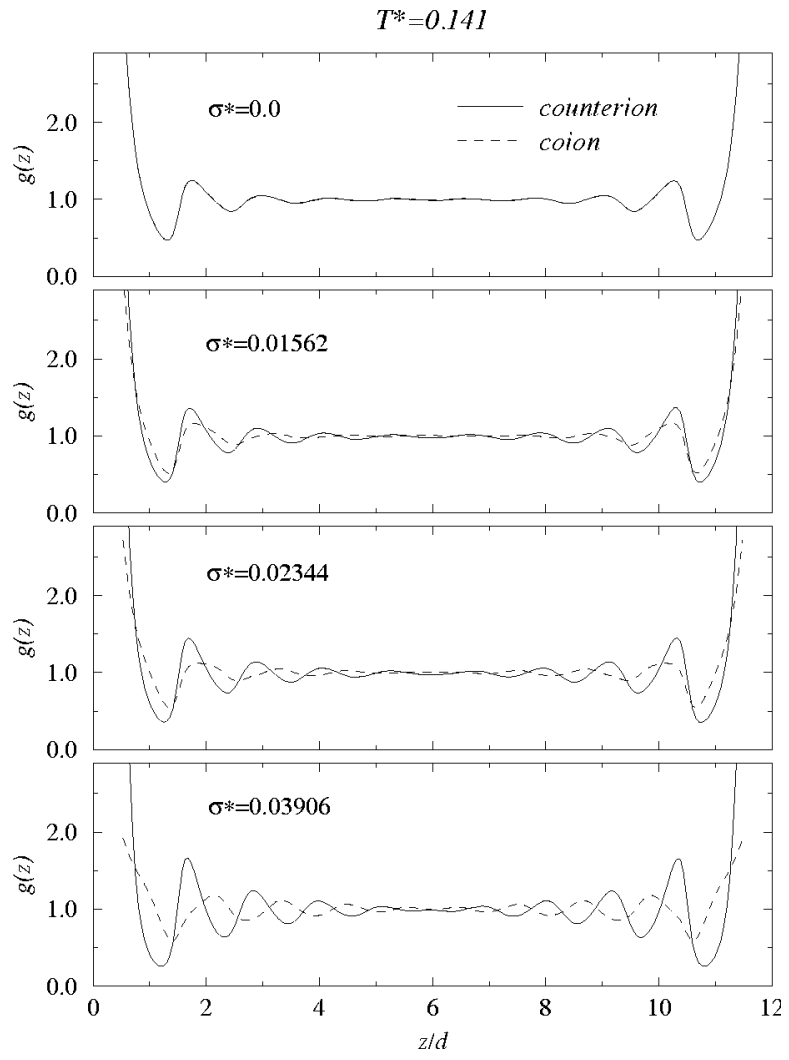

FIG. 1. Normalized density profiles, $g(z)=\rho(z) / \rho_{0}$, of a molten salt DL at $T^{*}=0.141$ and $\eta \sim 0.3503$ for various surface charges.

Figure 3(a) shows the potential as a function of $\sigma^{*}$. The potential is divided by $T^{*}$ so that the curves are separated and are seen more clearly. To a reasonable approximation, $\Psi_{0}^{*}$ is a linear function of $\sigma^{*}$. In Fig. 3(b) the MC and MSA capacitances are plotted as functions of $\sigma^{*}$. It is seen that at $T^{*}=0.282$ the MC capacitance seems to converge to a well

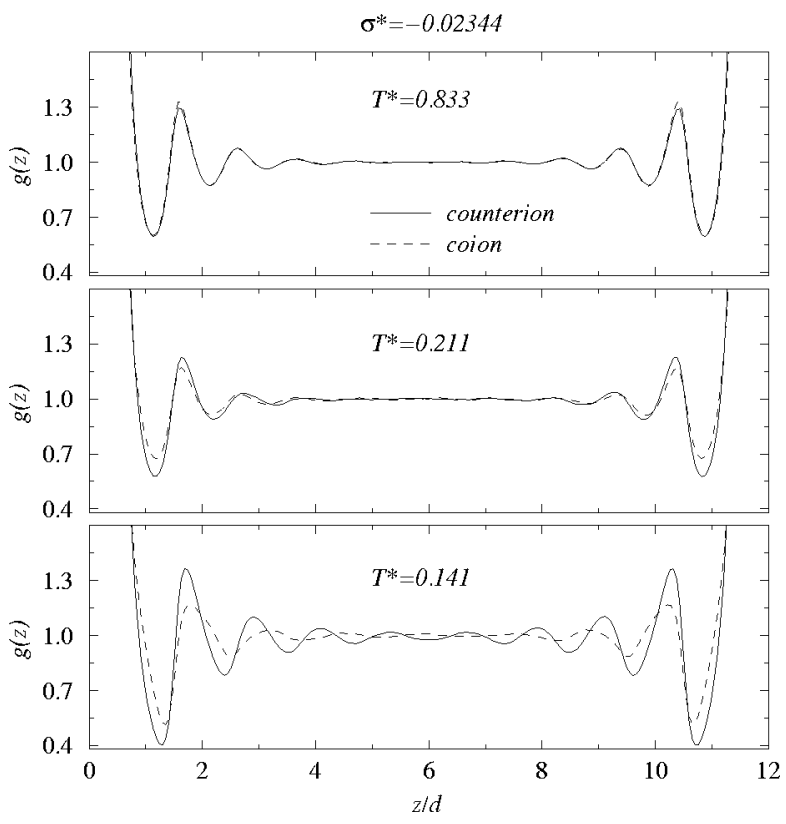

FIG. 2. Normalized density profiles, $g(z)=\rho(z) / \rho_{0}$, of molten salt DL at $\sigma^{*}=-0.02344$ and $\eta \sim 0.3503$ for various reduced temperatures. 


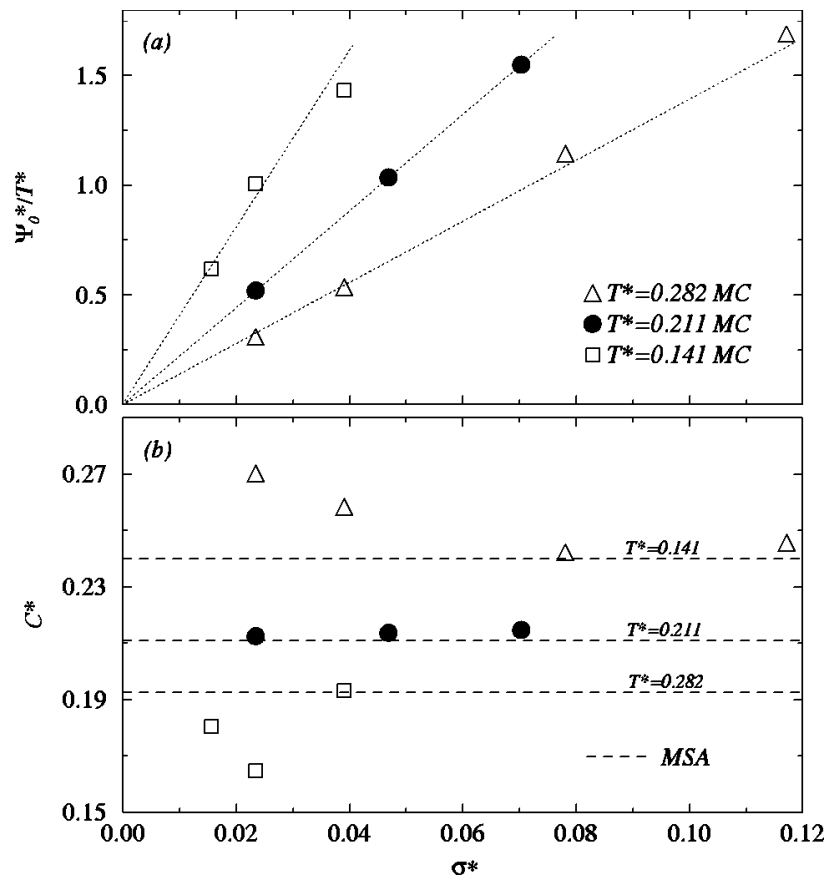

FIG. 3. Reduced potential (a) and capacitance (b) as functions of $\sigma^{*}$ for $\eta \sim 0.3503$ and three different reduced temperatures. The symbols give the $\mathrm{MC}$ simulation, in part (a) the dotted lines are given only as a guide to the eye, and in part (b) the dashed lines give the MSA data. The MSA data were obtained by using $\rho_{0}^{*}=0.64$ for the bulk density. Note that for the MC data in part (b), the temperature increases from bottom to top whereas the opposite is true for the MSA curves.

defined value as $\sigma^{*}$ goes to zero, and at $T^{*}=0.211$ it is nearly independent of $\sigma^{*}$. The data for $T^{*}=0.141$ shows more scatter perhaps because this temperature is close to the limit where the chains tend to form. Therefore, additional simulations were performed for $\sigma^{*}=-0.02344$ at various temperatures. We believe that this value of $\sigma^{*}$ is sufficiently small so that, to a reasonable approximation, we need not distinguish between the differential and integral capacitance. The integral capacitances that resulted were accepted as equal to the differential capacitances at the point of zero charge.

In Fig. 4 we have plotted $C^{*}$ as a function of $T^{*}$. We see that at low temperatures $C^{*}$ increases with increasing temperature (this behavior agrees with experimental results), while at higher temperatures $C^{*}$ decreases with increasing temperature, as do the MSA, GC, and GCS capacitances. Though error bars are not shown in the figure, the capacitances, we believe, have large (at least \pm 0.01 ) statistical errors. Nevertheless, neither this nor the question about whether the differential and integral capacitances are equal, should influence the fact that the capacitance increases with the increasing temperature if $T^{*} \leqslant 0.282$.

In Fig. 5 we show the charge [Fig. 5(a)], and the electrostatic potential profiles [Fig. $5(\mathrm{~b})]$ for the surface charge $\sigma^{*}=-0.02344$ at various temperatures. Note that at low temperatures there is charge inversion in the charge profiles. There is a layer of counterions near the electrode but there are alternating layers of coions and counterions at greater distances from the electrode. From these curves an explanation can be derived concerning the positive temperature de-

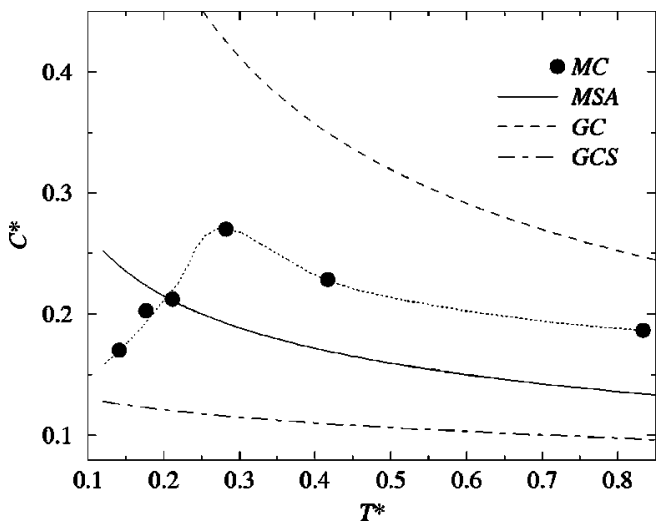

FIG. 4. Reduced capacitance as a function of the reduced temperature as obtained from MC simulations (symbols), and the MSA, GC, and GCS theories (curves) for $\eta \sim 0.3503$. The theoretical curves were obtained by using $\rho_{0}^{*}=0.64$ for the bulk density. The dotted line on the simulation data is intended only as a guide to the eye.

pendence of the capacitance. Since $\Psi_{0}^{*}-\Psi^{*}(d / 2)$ is the same for each curve due to the fixed surface charge, the behavior of the capacitance is determined by $\Psi^{*}(d / 2)$ [these are given by the open circles in Fig. 5(b)]. If $\Psi^{*}(d / 2)$ increases, then the magnitude of $\Psi_{0}^{*}$ decreases, and consequently $C^{*}$ increases. In our opinion, the increase in $\Psi^{*}(d / 2)$ at low temperature is a consequence of the strong charge inversion. The packing of the particles is dense, and a distinct layer of coions appears close to the wall at $z=d$. This results in a deep valley in the potential near $z=d$ as seen in Fig. 5(b). By increasing the temperature, the amplitude of the charge density profiles decreases, and the locations of the maxima and minima are shifted to the right. This means that the packing of the layers in the DL has become

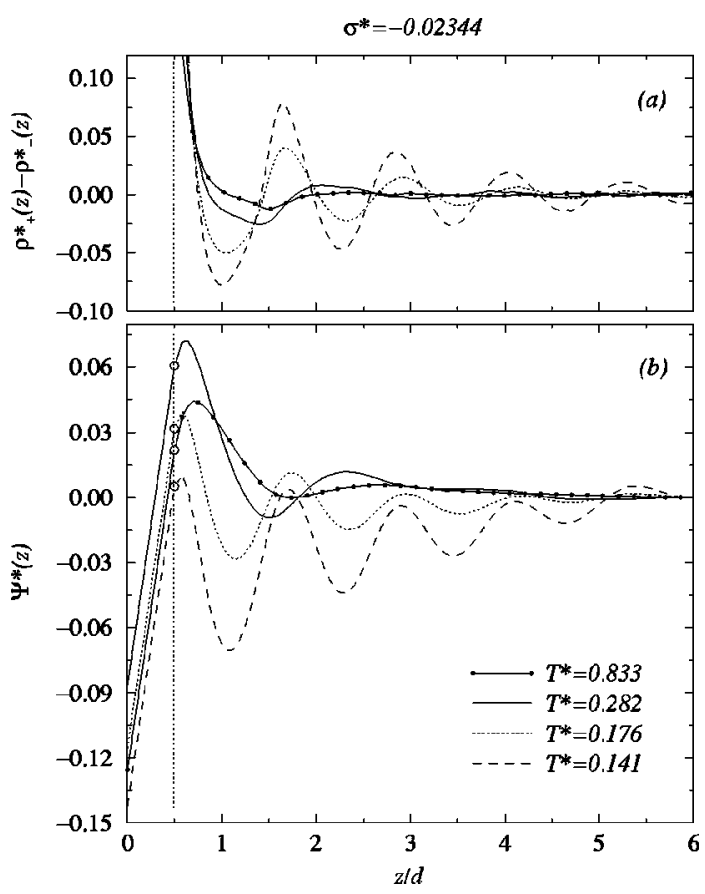

FIG. 5. The reduced charge distribution (a) and the reduced electrostatic mean potential profiles (b) at $\sigma^{*}=-0.02344$ for $\eta \sim 0.3503$ and various temperatures. 


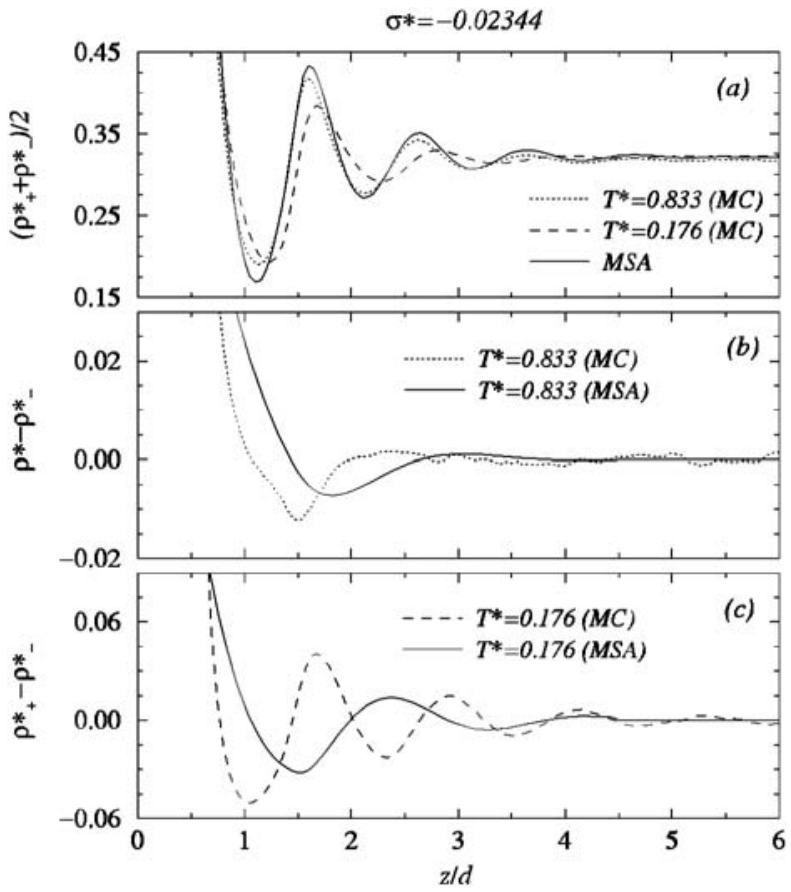

FIG. 6. The total density $(a)$, and the charge density profiles (b) and (c) for $\eta \sim 0.3503$ at $T^{*}=0.833$ and $T^{*}=0.176$ with a comparison of the $\mathrm{MC}$ simulation (dotted and dashed lines) and MSA (solid lines) results. In the case of the MSA the total density profile is independent of the temperature and is equal to the profile of hard spheres against a hard wall. Note the difference in scale between the $y$ axis of parts (b) and (c).

less dense. These result in potential curves that are shifted upwards with the increasing temperature, and eventually in the increase of $\Psi^{*}(d / 2)$ and $C^{*}$ with increasing temperature.

Above $T^{*}=0.282$ this behavior vanishes. The packing of the layers of the particles does not change too much, and the magnitude of the charge inversion decreases. This results in the decrease of $\Psi^{*}(d / 2)$ and $C^{*}$ with increasing temperature. Above $T^{*}=0.282$, the simulation and MSA capacitances are in rough agreement. The slopes of the simulation and MSA capacitances are in quite good agreement at the higher temperatures.

The MSA profiles that are compared to the simulation profiles in Fig. 6 show oscillations and charge inversion in qualitative agreement with our simulations. In contrast, the GC and GCS theories would not exhibit this behavior. The MSA contact values of the profiles are too small. In part this is due to the linearized nature of the MSA. In addition, in the MSA the layer of coions is further from the electrode than is the case in the simulations. As a result, the coions have less of an effect on the potential difference and capacitance than occurs in the simulations.

\section{SUMMARY AND CONCLUSIONS}

We found that if the temperature is low enough, the capacitance at the point of zero charge increases with increasing temperature. Unfortunately, we are unable to obtain results for the low values of the reduced temperature that characterize molten salts. Perhaps with a larger cell and longer runs we could get results for lower values of $T^{*}$. However, it does seem doubtful that we could attain values of $T^{*}$ that are lower by the factor of 10 needed for comparison with experiment. It seems likely that numerical theories, such as the hypernetted chain approximation would have convergence problems for such low $T^{*}$. We expect that second order theories, which tend to be more accurate, would have even greater problems.

At the lower values of $T^{*}$ for which we have obtained results, we found that the capacitance increases with increasing temperatures. Although we cannot be certain that this behavior persists to even lower $T^{*}$, it is at least plausible that this is the case. At very least, we have shown that positive values of $d C / d T$ are possible for the charged hard sphere model. This makes it likely that it is the approximations imposed by the MSA, GC, and GCS theories that fail for molten salts, and not the charged hard sphere model.

It is interesting that there is an inversion in $C$ versus $T$ at higher temperatures. At reduced temperatures $T^{*} \geqslant 0.282$, the behavior of $C$ is similar to that of dissolved salts, and the $\mathrm{GC}$ and MSA theories. This raises the interesting question of whether a similar inversion in the behavior of $C$ takes place at low densities. It is at least conceivable that at very low temperatures dissolved salts exhibit a capacitance near the point of zero charge that increases with increasing temperature.

\section{ACKNOWLEDGMENTS}

The research was supported by the National Science Foundation (Grant CHE96-01971), by the Hungarian National Research Fund (OTKA-D25516), by the donors of the Petroleum Research Fund, administered by the American Chemical Society (Grant No. ACS-PRF 31573-AC9), and the Research Grants Council of Hong Kong (HKU249/95P).

${ }^{1}$ K. R. Painter, P. Ballone, M. P. Tosi, P. J. Grout, and N. H. March, Surf. Sci. 133, 89 (1983).

${ }^{2}$ P. Ballone, G. Pastore, M. P. Tosi, K. R. Painter, P. J. Grout, and N. H. March, Phys. Chem. Liq. 13, 269 (1984).

${ }^{3} \mathrm{~S}$. Li and R. M. Mazo, J. Chem. Phys. 86, 5757 (1987).

${ }^{4}$ G. M. Torrie and J. P. Valleau, J. Chem. Phys. 73, 5807 (1980).

${ }^{5}$ B. Larsen, J. Chem. Phys. 65, 3431 (1976).

${ }^{6}$ D. Boda, K. Y. Chan, and D. Henderson, J. Chem. Phys. 109, 7362 (1998).

${ }^{7}$ B. Larsen and S. A. Rogde, J. Chem. Phys. 68, 1309 (1978). 